\title{
Bindung und Psychotherapie. Teil 2
}

\author{
Karin Pinter
}

Online publiziert: 31. Juli 2014

(C) Springer-Verlag Wien 2014

Das Forschungsinteresse an bindungsrelevanten Themen ist ungebrochen. Bis sich Erkenntnisse aus der Forschung in den verschiedenen gesellschaftlichen Bereichen zu einem selbstverständlichen Alltagswissen durchsetzen und anwendbar werden, braucht es einige Zeit. Bindung spielt nicht nur in der Entwicklungspsychologie, im Verstehen von Psychopathologien, in der Prävention wie Elternberatung, in allen Bereichen der Kinderpflege, -betreuung und in Erziehungseinrichtungen, sondern selbstverständlich auch in der Psychotherapie von Kinder, Jugendlichen, Erwachsenen und Paaren eine unverzichtbare Rolle.

Im zweiten Teil „Bindung und Psychotherapie“ finden sich Beiträge zu methodischen Instrumenten zur Erfassung des Bindungsstatus und daraus abgeleiteten Anwendungsstrategien in der psychotherapeutischen Behandlung bzw. in der präventiven Elternarbeit. Exemplarische Fallbeispiele veranschaulichen die forschungsund theoriebasierten Überlegungen.

Über die Verwendung des Adult Attachment Projective Picture Systems zur Diagnostik und psychoanalytischen Behandlung von Erwachsenen berichtet Buchheim, Gander und Juen. In der von den Autorinnen präsentierten Studie werden erstmals signifikante Veränderungen von Bindungstraumata bei chronisch depressiven PatientInnen nach 15-monatiger psychoanalytischer Behandlung nachgewiesen.

Zur Förderung der Sensibilisierung von Eltern gegenüber der Erlebensperspektive ihres Kindes entwickelt
Gloger-Tippelt und eine Forscherinnengruppe auf der Basis von Bindungsforschung und bindungsorientierten Instrumenten ein neues Modul, das sie ganz aktuell erproben und weiterentwickeln wollen. Die Autorinnen berichten über die ersten Erfahrungen damit und stellen die Vorbereitung eines Schulungskonzeptes für PsychotherapeutInnen in Aussicht.

An Hand einer Fallvignette aus ihrer Praxis beschreibt Topel die positiven Auswirkungen ihrer bindungsgeleiteten Behandlungsstrategien auf die Symptomatik eines Mädchens und auf die Veränderung des problematischen Elternverhaltens unter Anwendung des Mentalisierungskonzeptes. Topel diskutiert verschiedene theoretische Konzepte, die auf Bindungsforschung basieren.

Stärker konzeptorientiert erörtern Löffler-Stastka, Parth, Grassl, Karwautz und Lodermeier die Überschneidungen und Differenzen zwischen Objektbeziehungstheorie und Bindungstheorie. Sie orten großes Forschungspotential für zukünftige Forschung, wenn es gelingt, diese beiden Konzepte zu verbinden.

Als frei eingereichten Originalbeitrag zum Thema Unfruchtbarkeit stellt Asimakis verschiedene theoretische psychoanalytische Konzepte gegenüber und leitet daraus Fragestellungen ab, die für die psychotherapeutische Praxis relevant sein könnten. 